\title{
Fabrication of Nanoparticle Films Applying Directed Self-assembly
}

\author{
Akira Watanabe, Naoko Kihara, Takeshi Okino, and Ryousuke Yamamoto
}

\author{
Corporate Research \& Development Center, Toshiba Corporation \\ 1, Komukai-Toshiba-cho, Saiwai-ku, Kawasaki, 212-8582, Japan
}

\begin{abstract}
The fabrication process of large-area self-assembled monolayers of gold nanoparticles was investigated, focusing on the effect of the solvent applying dip-coating system at fast rates $(\mathrm{mm} / \mathrm{sec})$. The uniformity of the particle array formed on the substrate was improved by polymer coating on the nanoparticle surface that prevented movement of particles during the drying process. We have also examined the effects of solvent in suspension on formation of nanoparticle films. The uniformity of films on the substrate was improved by selecting methyl ethyl ketone (MEK) as a solvent. In addition, it was found that the solvent annealing with MEK vapor promoted rearrangement of nanoparticle alignment in the films. Self-assembling performances of nanoparticles were also demonstrated by applying topographical pattern.
\end{abstract}

Keywords: Nanoparticle, Dip-coating, Solvent annealing, Self-assembly

\section{Introduction}

Low-cost fabrication processes for large-area periodic nanostructures are required in production of battery electrodes [1,2], photonic crystal devices [3], magnetic recording media [4-6] and other nanostructure devices [7]. To form such nanostructures, a method for drawing a pattern on a resist using a drawing apparatus employing electron-beam or ultraviolet ray, a method using a self-assembling phenomena of block copolymers $[5,8,9]$ or particles $[1-3,6,10-16]$, and the like are used. In particular, particles containing metal elements have high etch selectivity sufficient for the etching mask application. A variety of well-controlled methods can be used to form ordered particle arrays, such as spin-coating [17], drop-casting, Langmuir Blodgett [10] and dip-coating $[7,11,12]$. In particular, dip-coating is a widely used technique for the formation of colloidal ordered patterns for 2D particle arrays [5,10-13]. The mechanism by which the dispersed particles are brought together and crystallized in the thin wetting films is convective assembly. For preparation of uniform particle films, an evaporation rate is known to be important [11]

In this study, thin structured nanoparticle layers were prepared by a dip-coating system, and the relationship between morphology of prepared films and solvent of the suspension was discussed. We have also demonstrated the alignment of nanoparticles with patterned template substrate.

\section{Experimental Section}

\subsection{Materials}

Spherical gold nanoparticles $(10 \mathrm{~nm}$ core diameter) capped with amine moiety were purchased from Daiken Chemical Co., Ltd. The nanoparticles were dispersed $10.3 \mathrm{mg} / \mathrm{ml}$ in hexane. Thiol-terminated polystyrene $(5.0 \mathrm{~kg} / \mathrm{mol}$ PS-SH) and hydroxyl-terminated PS (14.0 kg/mol PS-OH) were purchased from Polymer Source, Inc. Propylene glycol monomethyl ether acetate (PGMEA), methyl ethyl ketone (MEK), tetrahydrofuran (THF) and toluene were purchased form Wako Pure Chemical Industries Ltd. Hexane and ethanol were purchased from Kanto Chemical Co., Inc. All materials were used without further 


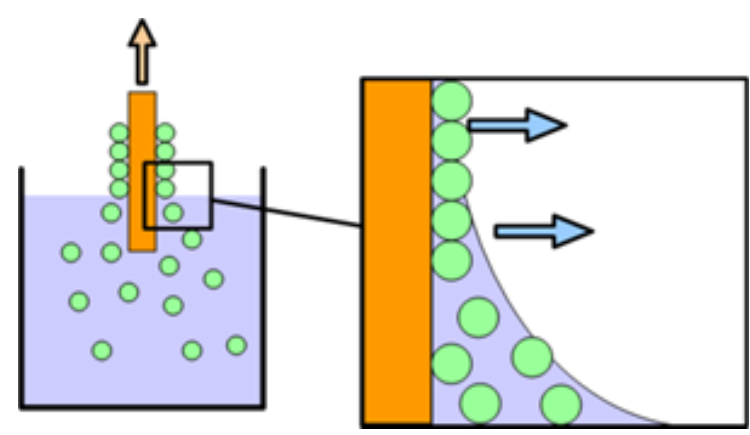

Figure 1. Schematic diagram of dip-coating mechanism for nanoparticle deposition.

purification.

\subsection{Sample Preparation}

\subsubsection{Treatment of Substrates}

For substrates, a silicon wafer or silicon wafer equipped with a trench pattern was used. A trench pattern with a width of $200 \mathrm{~nm}$ and a depth of 20 $\mathrm{nm}$ was fabricated using nanoimprint lithography and dry etching process based on a previously reported process [3]. On the $\mathrm{Si}$ surface, polystyrene brush layer was formed. On a substrate cleaned by exposure to deep-UV for 3 minutes, $0.5 \mathrm{wt} \%$ PGMEA solution of PS-OH was spin-coated. The substrates were then baked in vacuum at $170^{\circ} \mathrm{C}$ for 24 hours. Non-grafted PS-OH molecules on Si surface were eliminated by rinse with PGMEA.

\subsubsection{Grafting of PS-SH to the Gold Nanoparticles}

The purchased nanoparticles were capped with amine moiety as ligands. The modification of the particle surface was carried out as follows. To $10 \mathrm{~mL}$ of the suspended solution of gold nanoparticles capped with amine moiety, PS-SH in toluene $(1 \mathrm{~g} / 10 \mathrm{~mL})$ was added dropwise with constant stirring. The thiol-terminated PS chains reacted with the amine moiety on the particle surface. After stirring for 4 hours at room temperature, ethanol was added dropwise to the mixture for 5 minutes. The modified particles were collected by centrifugation at $5000 \mathrm{rpm}$ for 10 minutes and the supernatant was discarded.

\subsection{Process Flow of Experiment}

A schematic of the deposition mechanism of nanoparticles is shown in Figure 1. Dip coating of the colloidal nanospheres was performed using a dip-coating system (MD-0408-S5) purchased from SDI Company Ltd. The Si substrates whose surface was modified with PS-OH were fixed to a holder and vertically immersed in suspensions with a concentration of $2 \mathrm{wt} \%$ in a quartz cell. The substrates were then pulled up at a constant rate of $1.0 \mathrm{~mm} \mathrm{~s}^{-1}$ at room temperature. The experimental conditions were carefully chosen for homogeneous nanosphere deposition.

\subsection{Measurement}

Microstructures of the nanoparticle arrays were observed by scanning electron microscopy (SEM).

\section{Results and Discussion}

\subsection{Effect of polymer coating}

Figure 2 shows SEM images of the gold nanoparticle films on bare $\mathrm{Si}$ substrate. As shown in the SEM images, particles, which were capped with amine moiety or thiol-terminated PS, formed large-area monolayer array. In the Figure, white tone indicates the presence of gold nanoparticles. The amine-moiety-capped nanoparticles and PS-capped nanoparticles are dispersed in hexane and MEK, respectively.

The film of amine-moiety-capped nanoparticles shown in Fig. 2 (a) is observed to be a hexagonally close-packed lattice at short range but traces of cracking are evident in the layer, and the particles may have non-uniform pitch. On the other hand, as shown in Fig. 2 (b), PS-capped nanoparticles were arranged closely on a large scale without cracks. The particles are arranged closely to one another according to capillary force and Van der Waals force. While the film is drying, the particles may move, and cracking may occur in the particle layer during the drying process. Large-scale arrays are formed with steric barrier of PS that prevents particle movement during the drying process, but causes interparticle distance to increase. 

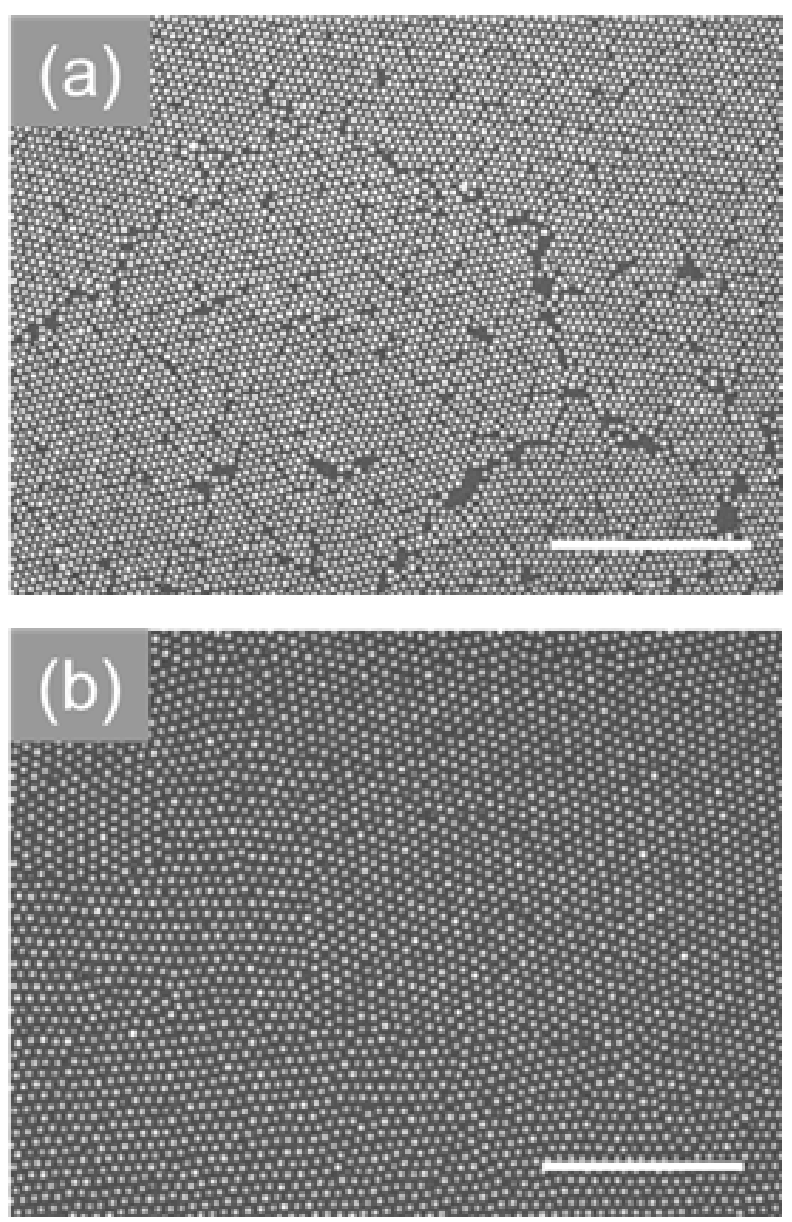

Figure 2. SEM images of each gold nanoparticle array. (a) amine-moiety-capped nanoparticles, (b) PS-capped nanoparticles. The scale bars indicate $300 \mathrm{~nm}$.

\subsection{Self-assembled without patterns}

The remarkable factor in the mechanism of self-assembling of suspended particles in wetting films is the volumetric flux balance of the evaporating solvent film and the assembling particles [6]. The evaporating solvents were interrelated in terms of boiling point, vapor pressure, temperature, humidity of the surrounding atmosphere, and interplay between solvent and particles. In this study, toluene, MEK, and THF


Figure 3. SEM images of gold nanoparticle array formed by several solvents in suspension. (a) THF, (b) MEK, (c) Toluene. The scale bars indicate $200 \mathrm{~nm}$.

were selected as the solvents for suspension from the viewpoint of drying rate. Table 1 shows the characteristics of the solvents. In each solvent, particles are dispersed completely.

Figure 3 shows SEM images of the films of particles suspended with various solvents. The arrangement of gold nanoparticle's position

Table 1. Characteristics of various solvents

\begin{tabular}{|c|c|c|c|}
\hline Solvent & $\begin{array}{c}\text { Boiling point } \\
\left({ }^{\circ} \mathbf{C}\right)\end{array}$ & $\begin{array}{c}\text { Vapor pressure } \\
\left(\mathbf{m m H h}, \mathbf{2 0}^{\circ} \mathrm{C}\right)\end{array}$ & $\begin{array}{c}\text { Solubility parameter } \\
\left(\mathrm{MPa} 1 / \mathbf{2}, \mathbf{2 0}{ }^{\circ} \mathrm{C}\right)\end{array}$ \\
\hline THF & $\mathbf{6 6}$ & 145 & $\mathbf{1 8 . 6}$ \\
\hline MEK & $\mathbf{7 9 . 5}$ & 95.3 & $\mathbf{1 9 . 0}$ \\
\hline Toluene & $\mathbf{1 1 0}$ & 22 & $\mathbf{1 8 . 2}$ \\
\hline
\end{tabular}


depends on the propensity of solvent. As shown in Fig. 3 (a), multi-layer and some voids are observed in the particle film formed by THF suspension. In the case of toluene as the solvent, various layers are observed (Fig. 3 (c)). In contrast, some defects are evident in the MEK suspension film as shown in Figure 3(b).

Dimitrov and Nagayama summarized the requirements for producing particle monolayers on substrate. According to their work, it is necessary to prevent the particle aggregation at the surface [6] by forming a wetting film of a thickness less than the particle diameter. Such condition is obtained when the solvent evaporation time is close to the particle arrangement time at the meniscus. Considering these facts, it seems reasonable to assume that THF causes formation of multi-layer particles on the substrate because of its high evaporation rate. On the other hand, it seems reasonable to assume that the low evaporation rate of toluene leads to formation of uneven wetting film in order to prevent drying at the edge of the meniscus, case multi-layer and some voids on the substrate.

The MEK suspension is suitable for the dip-coating system to form gold nanoparticles 10 $\mathrm{nm}$ in diameter on the substrate because the volumetric flux balance of the evaporating solvent film and the assembling particles is favorable. For formation of uniform particles on the substrate, selection of the optimal solvent with respect to the evaporation rate seems to be required.

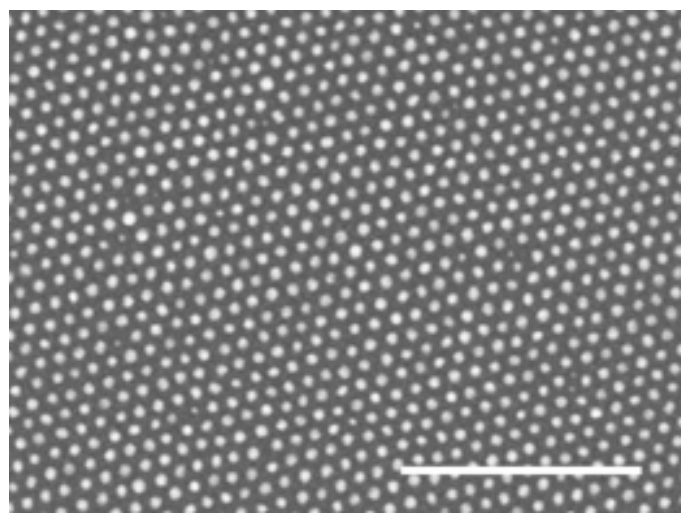

Figure 4. SEM images of gold nanoparticles array formed by MEK in suspension and followed by solvent annealing with MEK for $2 \mathrm{~h}$. The scale bar indicates $200 \mathrm{~nm}$.

\subsection{Effect of solvent annealing}

Solvent annealing is known to be one of the techniques for ordering the self-assembly patterns in the research field of block copolymers [4]. The block copolymer is exposed to a solvent vapor under ambient temperature to enhance the mobility of polymers. We applied this rearrangement effect for alignment of particles. For a sample suitable for solvent annealing, the film formed by MEK solvent was used.

In view of the desirability of using the same solvent for dispersion, MEK was the solvent selected for the process. Figure 4 shows SEM images of samples after solvent annealing with MEK vapor. After 2 hours of exposure to MEK vapor, gold nanoparticles were observed with well-ordered alignment compared with Figure 3(b). This result indicates that particles are rearranged in solvent annealing. A region of single-domain particles was expanded to one of the order of several micrometers by solvent annealing.
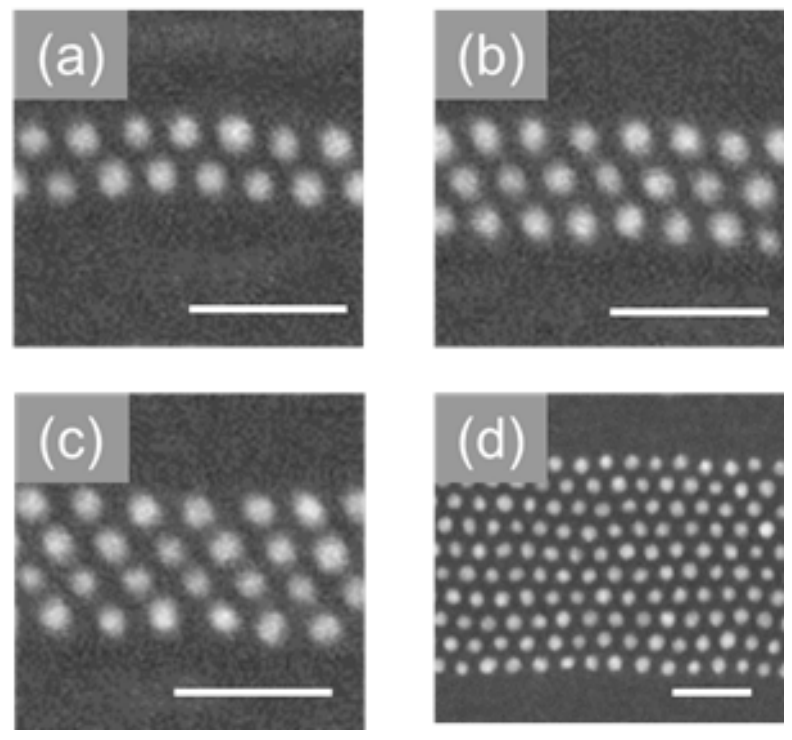

Figure 5. SEM image of gold nanoparticle array with topographically patterned templates with different widths: (a) $30 \mathrm{~nm}$, (b) $45 \mathrm{~nm}$, (c) $60 \mathrm{~nm}$, (d) $150 \mathrm{~nm}$. The scale bars indicate $50 \mathrm{~nm}$.

After solvent annealing, the size of the regions of the single-domain particles expands from hundreds nanometers to several micrometers. It is seems that the domain was expanded during solvent annealing. 


\subsection{Self-assembled with a template}

In trench patterns, we observed the ordering of nanoparticles as shown in Figure 5. The number of rows that could fit between the guidelines depended strongly on the spacing of the lines. The natural lattice parameter of nanoparticle film was measured to be $\sim 17( \pm 1) \mathrm{nm}$ on average (Figure 4). Two to ten rows could be obtained by changing the grating width from $30 \mathrm{~nm}$ to $150 \mathrm{~nm}$. It seems that directed self-assembly of nanoparticles is possible in the same way as that of block copolymers.

\section{Conclusion}

Self-assembly of gold nanoparticles was investigated by using a dip-coating system at fast rates. The uniformity of aligned nanoparticle array was strongly dependent on the evaporation rate of solvent. In the case of gold nanoparticles $10 \mathrm{~nm}$ in diameter, a uniform film was fabricated by selecting MEK as the optimal solvent in terms of its evaporation rate. Moreover, solvent annealing with MEK vapor yielded rearrangement of nanoparticles on the films. We have also demonstrated the alignment of nanoparticles with patterned substrate. The possibility of highly uniform alignment of the particles in width-controlled multiple rows was demonstrated. Future work will focus on improving larger-area ordering by controlling both evaporation rate and width of trench patterns.

\section{References}

1. J. S. Sakamoto, B. Dunn, J. Mater. Chem., 12 (2002) 2859-2861.

2. H. Xhang, S.Yu, P. V. Braun, Nat. Nanotechnol. 6 (2011) 277.

3. E. Armstrong, W. Kjunsin, M. Osiak, M. Blomker, C. M. Sotomayor Torres, and C. O’Dwyer, Small, 10 (2014) 1895.
4. Jonathan A. Fan, Chihhui Wu, Kui Bao, Jiming Bao, Rizia Bardhan, Naomi J. Halas, Vinothan N. Manoharan, Peter Nordlander, Gennady Shvets, Federica Capasso, Sience, 328 (2010) 1135.

5. H. Hieda, Y, Yanagita, A, Kikitsu, T. Maeda and K. Naito, J. Photopolym. Sci. Technol., 19 (2006) 425.

6. H. Oshima, H. Tamura, M. Takeuchi, A. Inomata, Y. Yanagida, N. Matsushita, H. Komoriya, T. Uzumaki and A. Tanaka, Nanotechnology, 20 (2009) 455303.

7. Q. B. Meng, Z. Z. Gu, O. Sato and A. Fujishima, App. Phys. Lett., 77 (2000) 4313.

8. K. Naito, H. Hieda, M. Sakurai, Y. Kamata and K. Asakawa, IEEE TRANS. ON MAG., 38 (2002) 1949.

9. N. Sasao, R. Yamamoto, N. Kihara, T. Shimada, A. Yuzawa, T. Okino, Y. Ootera, Y. Kawamonzen, H. Hieda, T. Maeda, Y. Kamata and A. Kikitsu, $J$. Photopolym. Sci. Technol., 25 (2012) 27.

10. T. Wen and S. A. Majetich, ACS NANO, 5 (2011) 8868.

11. A. S. Dimitrov and K. Nagayama, Langmuir, 12 (1996) 1303.

12. B. G. Prevo and O. D. Velev, Langmuir, 20 (2004) 2099.

13. M. Asbahi, K. T. P. Lim, F. Wang, H. Duan, N. Thiyagaraiah, V. Ng and J. K. W. Yang, Langmuir, 28 (2012) 16782.

14. H. Y. Lelivre, J. Desbiens, and A. M. Ritcey, Langmuir 23 (2007) 2843.

15. K. Ohno, T. Morinaga, S. Takeno, Y. Tsujii, and T. Fukuda, Macromolecules 40 (2007) 9143.

16. N. N. Khanh and K. B. Yoon, J. Am. Chem. Soc., 131 (2009) 14228.

17. M. Pichumani, P. Bagheri, K. M. Poduska, W. G. Vinas, and A. Yethiraj, Soft Matter, 9 (2013) 3220 . 